\title{
Characterization of Failure Mechanism in Composite Materials Through Fractal Analysis of Acoustic Emission Signals ${ }^{\S}$
}

\author{
F. E. Silva ${ }^{a}$, L. L. Gonçalves ${ }^{a, b \dagger}$, D. B. B. Fereira ${ }^{c}$ and J. M. A. Rebello ${ }^{d}$ \\ ${ }^{a}$ Programa de Mestrado em Engenharia e Ciência de Materiais \\ Universidade Federal do Ceará, \\ Campus do Pici, Bloco 714, 60455-760 Fortaleza, CE, Brazil \\ ${ }^{b}$ Departamento de Fisica Geral, Universidade de São Paulo, \\ C.P. 66318 , 05315-970, São Paulo, SP, Brazil \\ ${ }^{c}$ Fundação de Apoio à Escola Técnica do Rio de Janeiro \\ 21311- 280 Rio de Janeiro, RJ, Brazil \\ ${ }^{d}$ Departmento de Engenharia Metalúrgica e de Materiais \\ Universidade Federal do Rio de Janeiro \\ CP 68505, 21945-970 Rio de Janeiro, RJ, Brazil
}

\begin{abstract}
In this paper it is presented a detailed numerical investigation of acoustic emission signals obtained from test samples of fibreglass reinforced polymeric matrix composites, when subjected to tensile and flexural tests. Various fractal indices, characteristic of the signals emitted at the different structural failures of the test samples and which satisfy non-stationary distributions, have been determined. From the results obtained for these indices, related to the Hurst analysis, detrended fluctuation analysis, minimal cover analysis and to the boxcounting dimension analysis, it has been shown they can discriminate the different failure mechanisms and, threfore, they constitute their signature.
\end{abstract}

${ }^{\dagger}$ Corresponding author.

E-mail: lindberg@fisica.ufc.br

On sabbatical leave from:

Departamento de Fisica

Universidade Federal do Ceará

Campus do Pici, Caixa Postal 6030

60451-970 Fortaleza, Ceará, Brazil

$\S$ Work partially financed by the Brazilian agencies CNPq, Finep (CT-Petro) and Capes. 


\section{INTRODUCTION}

In a recent paper Ferreira et al [1] discuss the characterization of failure mechanisms that occur in fibreglass reinforced polymeric matrix composites when subjected to tensile and flexural loads. The characterization was based on the analysis of acoustic emission signals emitted by the composite during the process of failure, which constitutes one the most important non-destructive testing for the detection of structural flaws in composite materials [2-5].

The samples studied were manufactured with E-glass fibre roving reinforced DER 331 epoxy resin and its preparation and experimental conditions are described in detail in ref. [1]. Besides tensile tests, flexural tests at three- and four-points were also applied and four failure modes have been observed, namely, matrix cracking, fibre braking, fibre/matrix debonding and delamination.

The main purpose of the study was to find the signature of these failure modes in the acustic emission signals. In order to identify these signatures, the signals were studied by using Fourier spectral analysis and wavelet analysis. Although relevant information has been obtained from these analyses, the authors in ref. [1] have not been able to characterize in a clear way the various failure mechanisms.

Therefore, in this paper we readdress the problem by looking at some fractal properties of the acoustic emission signals. In particular, we obtain the fractal indices related to the Hurst analysis [6], detrended fluctuation analysis [7], minimal cover analysis[8] and to the boxcounting dimension analysis [9], which will be used to characterize the different failure modes.

These types of analysis have been widely used in the study of random nonstationary series ranging from seismic [10] and climate data, [11] to wind speed [12] and financial data [13], and in the study of different music genres [14]. Their use in the characterization of acoustic signal has been introduced by Duta and Barat [15] in the analysis of ultrasonics backscattered signals obtained in the study of single crystal and polycrystalline materials. More recently, Matos et al. [16] have used this approach to characterize the ultrasonics backscattered signals obtained in the study of the cast iron with lamellar, vermicular and spheroidal microstructures.

The study presented in this paper extends the above mentioned analyses to a new type of acoustic signals, namely, the ones obtained in the acoustic emission nondestructive testing. The main objective of the work is to show that the parameters determined from these analyses can characterize the failure mechanisms in composite studied. To this aim and in order to establish the parameters to be calculated, we present in section 2 a brief review of the numerical analysis used in the treatment of the data, and in section 3 we present and discuss the results obtained. 


\section{NUMERICAL ANALYSIS}

The numerical treatment of the signals will be performed on data from Ascan, which contains the amplitude of the acoustic emission signals as a function of time. The parameters to be determined, as pointed out in the introduction, will be obtained from the Hurst analysis ( $\mathrm{R} / \mathrm{S}$ analysis) [6], detrended fluctuation analysis (DFA analysis) [7], minimal cover analysis [8] and boxcounting analysis [9].

In order to make the paper self-contained and to introduce the notation, we will present a brief review of the these numerical techniques which will be used in the analysis of the temporal series. They will be identified as the set of random values $\left\{y_{i}\right\}$, where the label $i$ corresponds to the time variable, which satisfy nonstationary distributions.

\subsection{Hurst analysis}

The $\mathrm{R} / \mathrm{S}$ analysis will provide information on the temporal correlations, on various time-scales, of the data. Given the temporal series $\left\{y_{i}\right\}$, with $N$ terms $(1 \leqslant i \leqslant N)$, we define the average in the interval $n$ as

$$
<y>_{n}=\frac{1}{n} \sum_{i=1}^{n} y_{i},
$$

and the accumulated deviation from the mean as

$$
Y(j, n)=\sum_{i=1}^{j}\left(y_{i}-<y>_{n}\right),
$$

where $n$ varies from 2 to $N$.

From these results, we can also define in the interval $n$ the range $R(n)$ of the accumulated deviation in the form

$$
R(n)=\max _{1 \leqslant j \leqslant n} Y(j, n)-\min _{1 \leqslant j \leqslant n} Y(j, n),
$$

and the standard deviation $S(n)$ as

$$
S(n)=\sqrt{\frac{\sum_{j}^{n}\left(y_{j}-<y>_{n}\right)}{n} .}
$$

Finally, we can obtain the rescaled range $R(n) / S(n)$ which should satisfy the scaling relation

$$
\frac{R(n)}{S(n)} \sim n^{H},
$$


where $H$ is the Hurst exponent [6].

In the scaling regime, the previous expression can be written as

$$
\frac{R(n)}{S(n)}=A_{H} n^{H},
$$

which defines the amplitude $A_{H}$. Although this parameter has no universal characteristic, as the amplitudes in the scaling laws in critical phenomena [17] where they are related to the interactions, they can be used however as an additional parameter to characterize the temporal series.

\subsection{Detrended fluctuation analysis}

The DFA analysis [7] aims to study the temporal correlations by eliminating the spurious trends in the data which can conduct to misleading results. The method consists initially in obtaining a new integrated temporal series $\left\{z_{i}\right\}$, from the original one $\left\{y_{i}\right\}$, given by

$$
z_{j}=\sum_{i=1}^{j}\left(y_{i}-<y>\right),
$$

where the average $\langle y\rangle$ is defined as

$$
<y>=\frac{1}{N} \sum_{i=1}^{N} y_{i} .
$$

In the following step the series is divided in time intervals of width $n$, and an order- $l$ polynomial is fitted in each interval, and we identify the analysis as DFA- $l$. Then, the detrended variation function of order $l$ in the interval $j, \Delta_{j}^{l}(j)$, is obtained by subtracting the local trend contained in the fitted polynomial, and is given by

$$
\Delta_{j}^{l}(n)=\sum_{i=(j-1) n+1}^{j n}\left(z_{i}-z_{i}^{l}\right)^{2},
$$

where $z_{i}^{l}$ is the value from the fitted polynomial.

Finally, we calculate the mean root square fluctuation $F^{l}(n)$

$$
F^{l}(n)=\sqrt{\frac{1}{N} \sum_{j=1}^{\text {int }[N / n]} \Delta_{j}^{l}(n),}
$$

which should scale as 


$$
F^{l}(n) \sim n^{\alpha},
$$

where $\alpha$ is the scaling exponent.

The detrended fluctuation analysis that we will present will be restricted to the linear case, namely, DFA-1. As in the case of the R/S analysis, eq.(11) can be written in the scaling regime as

$$
F^{l}(n)=A_{\alpha} n^{\alpha},
$$

which also defines a new characteristic parameter $A_{\alpha}$.

\subsection{Minimal cover analysis}

This method has been recently introduced [8], and it relates the minimal area necessary to cover a given plane curve, in a specified scale, to a power law behaviour. The scale is introduced by dividing the domain of definiton of the function in $n$ intervals of width $\delta$. In each interval $j(1 \leqslant j \leqslant n)$ we can associate a rectangle of base $\delta$ and height $A(j)$ defined as

$$
A_{j}=\max \left\{y_{i}, i \epsilon[j, j+\delta]\right\}-\min \left\{y_{i}, i \epsilon[j, j+\delta]\right\},
$$

such that the minimal area will be given by

$$
S(\delta)=\sum_{j=1}^{n} A_{j} \delta .
$$

In the scaling region, $S(\delta)$ should behave as

$$
S(\delta) \sim \delta^{2-D_{\mu}},
$$

where $D_{\mu}$ is the minimal cover dimension, which is equal to 1 when the curve presents no fractality. We can also define a new exponent $\mu$ given by

$$
\mu=D_{\mu}-1,
$$

which measures the fractality of the curve and satisfies the scaling relation

$$
V(\delta) \sim \delta^{-\mu},
$$


where $V(\delta)$ is the summmation of the heights of the rectangles

$$
V(\delta)=\sum_{j=1}^{n} A_{j} .
$$

The amplitude $A_{\mu}$, as in the previous cases, is defined in the expression

$$
V(\delta)=A_{\mu} \delta^{-\mu},
$$

and it also constitutes a new characteristic parameter.

\subsection{Boxcounting analysis}

The boxcounting dimension, which is one of the best known fractal dimension [9], is easily defined and obtained numerically. It can be introduced in a general d-dimensional euclidean space, where a hyper-volume is embedded, by considering the number of hypercubes of side length $\delta, \mathcal{N}(\delta)$, necessary to cover the entire volume. As $\delta \rightarrow 0, \mathcal{N}(\delta)$ satisfies the scaling relation

$$
\mathcal{N}(\delta) \sim \delta^{D_{B}},
$$

where $D_{B}$ is the boxcounting fractal dimension.

For non-fractal objects, this dimension corresponds to the topological dimension and, in particular, for continuous planar curves $D_{B}$ is equal to 1 .

The amplitude $A_{B}$ of the scaling relation is, in this case, given by

$$
\mathcal{N}(\delta)=A_{B} \delta^{D_{B}},
$$

and it also constitutes a new characteristic parameter. 


\section{RESULTS AND DISCUSSIONS}

A detailed description of the experimental setup and samples used for the acquisition of the acoustic emission signal from the different tests is presented by Ferreira et al. [1]. Besides the tensile test, the samples were submitted to three- and four-point flexural tests. They have identified four basic failure modes, namely, matrix cracking, fibre breaking, fibre/matrix debonding and delamination. In the different tests, the failure mechanisms were a result of a combination of these failure modes, and they are presented in Table 1. We also present in this Table the acronyms for the different specimens.

In order to reduce the noise in the data, the signals have been processed with an adjacent low-pass filter with five points. For each type of specimen the tests were carried out in 03 samples, which correspond to the number of signals available for each kind of mechanical failure.

In Figs. 1-4 we present the various analyses made in a given signal from the TEM specimen..These analyses are representative of the results obtained in the study of the other signals. In the Hurst analysis, Fig. 1, the crossover from shortto long- time correlations is always present. As can be seen in Figs. 2-4, this crossover also exists in the box counting analysis and in minimal cover analysis, but not in the DFA analysis. It should be noted that the presence existence of this crossover on the fractal analysis is characteristic of a multifractal behaviour.

Besides the eight parameters $H, A_{H}, \alpha, A_{\alpha}, \mu, A_{\mu}, D_{B}, A_{B}$, we can yet define an additional one which corresponds to the standard deviation $\sigma$ of the signal. This parameter has been recently introduced in the context of the characterization of climate of different regions in the United States from the analysis of maximum daily temperature time series [11].

From what we have presented, this multi-dimensional space parameter can be used to discriminate the various types of mechanical failure. As it will be shown in the figures relating the characteristic parameters, there is not a unique signature, since different combinations of the indices can lead to the identification of the signals. We have restricted our study to subspaces of the parameter space, as they can provide the desired signature of the signals. Explicitly we will consider the exponents $H, \alpha, \mu$ and $D_{B}$ as functions of the standard deviation of the signal, $\sigma$, and also as functions of the logarithm of its respective amplitude A.

These functions, which correspond to projections of the points of the parameter space in different planes, are shown in Figs. 5-17. Even considering that the data for each type of specimen consisted of three samples only, which is a poor statistical sampling, we have calculated the standard deviation of the variables presented as the error bar on these figures. From the analysis of these figures we can verify that the first discrimination attained is the separation of the failures caused by traction from the ones caused by flexion. This discrimination is clearly seen in the diagrams $H_{1} \times \sigma, H_{2} \times \sigma, D_{B 2} \times \sigma$ and $\alpha \times \sigma$ which are presented in the Figs. 5, 6, 8 and 11, respectively. This separation is the easiest to be obtained since the stress distributions in the samples are very different in the two cases, and this has strong effect on the acoustic emission signals.

By starting from any of these diagrams we can obtain the complete discrim- 
ination of all the failures mechanisms and even distinguish the results obtained from the three- and four-point flexural tests. This can be achieved by using the complete set of figures, namely, Figs. 5-17. In terms of these diagrams, all possible solutions of the problem are presented in the tree type graph shown in Fig. 18. In the branches of the tree, we designate the various failure modes and, in the nodes, we show the different two-dimensional diagrams which can lead to the desired discrimination. As can be seen on the solution tree, we can identify unmistakably all the failure modes from the acoustic emission signals and, moreover, show that there are multiple paths which lead to the identification we are looking for. 


\section{REFERENCES}

[1] Ferreira DB, Silva RR da, Rebello JMA, Siqueira MHS. Failure mechanism characterization in composite materials using spectral analysis and the wavelet transform of acoustic emission signals. Insight 2004; 46: 282-9.

[2] McIntire P, editor. Nondestructive Testing HandbookAcoustic Emission, v. 5, 2nd edition. Columbus: ASNT; 1987.

[3] Wegman RF. Nondestructive Test Methods for Structural Composites. SAMPE Handbook Series.Corvina: SAMPE; 1989.

[4] Summerscales J. NDT of advanced composites - an overview of the possibilities, British Journal of Non-destructive Testing 1990; 32 : 568-577.

[5] Clarke DJ. Thoughts on NDT for composites in the field. Insight 1995; 37: 938-939.

[6]. Hurst HE. Long-term capacity of reservoirs. Trans. Am. Soc. Civ. Eng. 1951; 116: 770-808.

[7]. Peng CK, Buldyrev V, Havlin S, Simmons M, Stanley HR, Goldberger AL. Mosaic organization of DNA nucleotides. Phys. Rev. E 1994; 49: 1685-1689.

[8] Dubovikov MM, Starchenko NV, Dubovikov MS. Dimension of minimal cover and fractal analysis of time series. Physica A 2004; 339: 591-608.

[9] See e.g. Addison PS. Fractals and Chaos. London: IOP; 1997.

[10] Telesca L, Lapenna V, Macchiato M. Mono- and multi-fractal investigation of scaling properties in temporal patterns sequences of seismic sequences. Chaos, Solitons \& Fractals 2004; 19: 1-15.

[11] Kurnaz ML. Detrended fluctuation analysis as a statistical tool to monitor the climate. J. Stat. Mech.: Theor. Exp. 2004; P07009.

[12] Govindan RB, Kantz H. Long-term correlations and multifractality in surface wind speed. Europhys. Lett. 2004; 68: 184-190.

[13] Carbone A, Castelli G, Stanley HE. Time-dependent Hurst exponent in financial time series. Physica $A$ in press.

[14] Jennings HD, Ivanov P Ch, da Silva PC, Viswanathan GM. Physica A 2004; 336: 585-594.

[15] Dutta D, Barat P. J. Acoust. Soc. Am. 1995; 98: 938-942.

[16] Matos JMO, de Moura EP, Küger SE, Rebello JMA. Chaos, Solitons 83 Fractals 2004; 19: 55-60.

[17] See e.g. Plischke M, Bergensen B. Equilibrium Statistical Physics. Singapore: World Scientific; 1994. 
Table 1. Failure modes and respective mechanical tests for the different specimen types.

\begin{tabular}{|c|c|c|}
\hline Test & Specimen type & Failure modes \\
\hline Tensile & Epoxy (TME*) & Matrix cracking \\
\hline Tensile & Fibre/Epoxy(TLEV*) & $\begin{array}{c}\text { Fibrebreaking } \\
\text { Matrixcracking } \\
\text { Fibre/matrixdebonding }\end{array}$ \\
\hline Tensile & Fibre/Epoxy(TTEV*) & Matrix cracking \\
\hline 4-point flexural & Fibre/Epoxy(F41*) & $\begin{array}{c}\text { Fibrebreaking } \\
\text { Matrixcracking } \\
\text { Fibre/matrixdebonding }\end{array}$ \\
\hline 4-point flexural & Fibre/Epoxy(F41S*) & $\begin{array}{c}\text { Fibrebreaking } \\
\text { Matrixcracking } \\
\text { Fibre/matrixdebonding }\end{array}$ \\
\hline 4-point flexural & Fibre/Epoxy(F42*) & $\begin{array}{c}\text { Fibrebreaking } \\
\text { Matrixcracking } \\
\text { Fibre/matrixdebonding } \\
\text { Delamination }\end{array}$ \\
\hline 3-point flexural & Fibre/Epoxy(F31*) & $\begin{array}{c}\text { Fibrebreaking } \\
\text { Matrixcracking } \\
\text { Fibre/matrixdebonding }\end{array}$ \\
\hline 3-point flexural & Fibre/Epoxy(F31S*) & $\begin{array}{c}\text { Fibrebreaking } \\
\text { Matrixcracking } \\
\text { Fibre/matrixdebonding }\end{array}$ \\
\hline 3-point flexural & Fibre/Epoxy(F41*) & $\begin{array}{c}\text { Fibrebreaking } \\
\text { Matrixcracking } \\
\text { Fibre/matrixdebonding } \\
\text { Delamination }\end{array}$ \\
\hline \multicolumn{3}{|c|}{${ }^{*}$ Specimen acronyms. } \\
\hline & & face tres \\
\hline
\end{tabular}




\section{Figure captions}

Fig. 1- Hurst analysis of a signal obtained from a TME specimen.

$H_{1}$ and $H_{2}$ are the Hurst exponents associated with short- and long-time correlations, respectively.

Fig. 2- DFA analysis for the signal used in Fig. 1.

Fig. 3- Minimal cover analysis for the signal used in Fig. 1. $\mu_{1}$ and $\mu_{2}$ are the variation indices associated with large and small fractal scales, respectively.

Fig. 4- Box counting analysis for the signal used in Fig. 1.

$D_{B 1}$ and $D_{B 2}$ are the box counting dimensions associated with large and small fractal scales, respectively.

Fig. 5- Hurst exponent $H_{1}$ (short-time correlations) as a function of the standard deviation $\sigma$ of the signal.

Fig. 6- Hurst exponent $H_{2}$ (long-time correlations) as a function of the standard deviation $\sigma$ of the signal.

Fig. 7- Box counting dimension $D_{B 1}$ (large fractal scale) as a function of the standard deviation $\sigma$ of the signal.

Fig. 8- Box counting dimension $D_{B 2}$ (small fractal scale) as a function of the standard deviation $\sigma$ of the signal.

Fig. 9- Variation index $\mu_{1}$ (large fractal scale) as a function of the standard deviation $\sigma$ of the signal.

Fig. 10- Variation index $\mu_{2}$ ( small fractal scale) as a function of the standard deviation $\sigma$ of the signal.

Fig. 11- DFA exponent $\alpha$ as a function of the standard deviation $\sigma$ of the signal.

Fig. 12- Hurst exponent $H_{1}$ (short-time correlations) as a function of the logarithm of the amplitude $A_{H 1}$.

Fig. 13- Hurst exponent $H_{2}$ (long-time correlations) as a function of the logarithm of the amplitude $A_{H_{2}}$.

Fig. 14- Box counting dimension $D_{B 1}$ (large fractal scale) as a function of the logarithm of the amplitude $A_{D_{B 1} 1}$.

Fig. 15- Box counting dimension $D_{B 2}$ (small fractal scale) as a function of the logarithm of the amplitude $A_{D_{B 1}}$.

Fig. 16- Variation index $\mu_{1}$ (large fractal scale) as a function of the logarithm of the amplitude $A_{\mu 1}$.

Fig. 17- Variation index $\mu_{2}$ (small fractal scale) as a function of the logarithm of the amplitude $A_{\mu 2}$.

Fig. 18 - Tree summarizing all possible solutions for the discrimination of the various failure modes, which correspond to different paths on the complete graph. The modes are shown on the branches, and the diagrams discriminating the modes are attached to the nodes. 


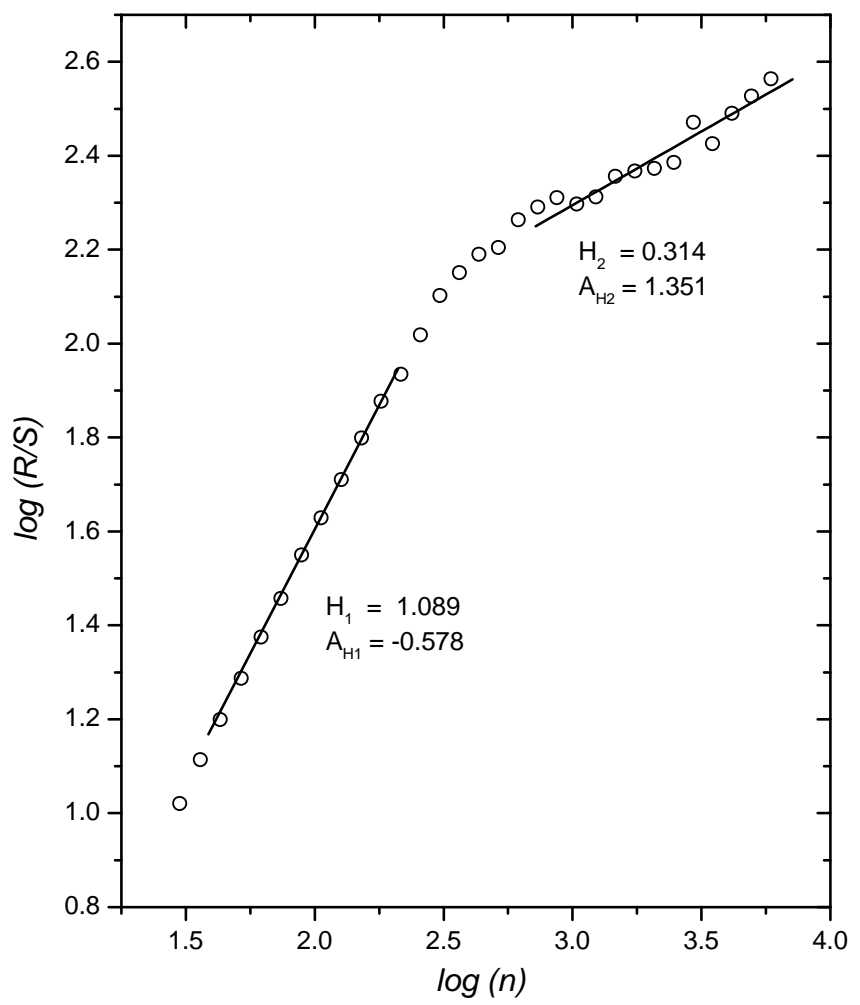

Fig. 1 


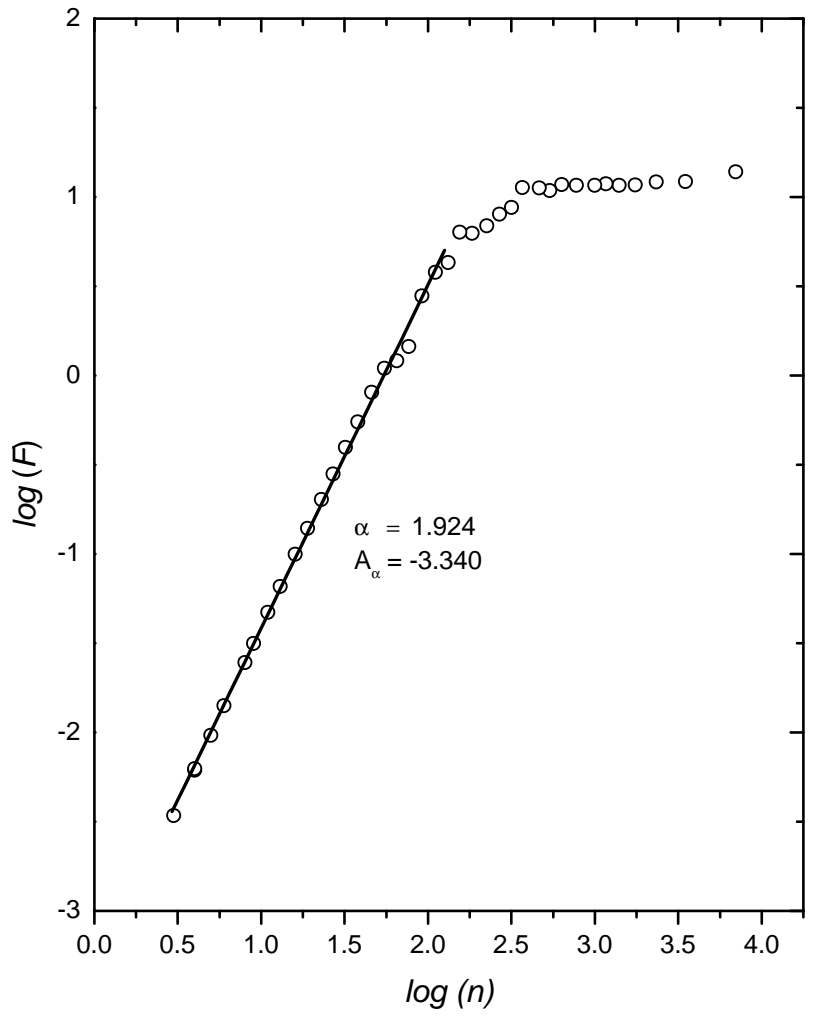

Fig. 2 


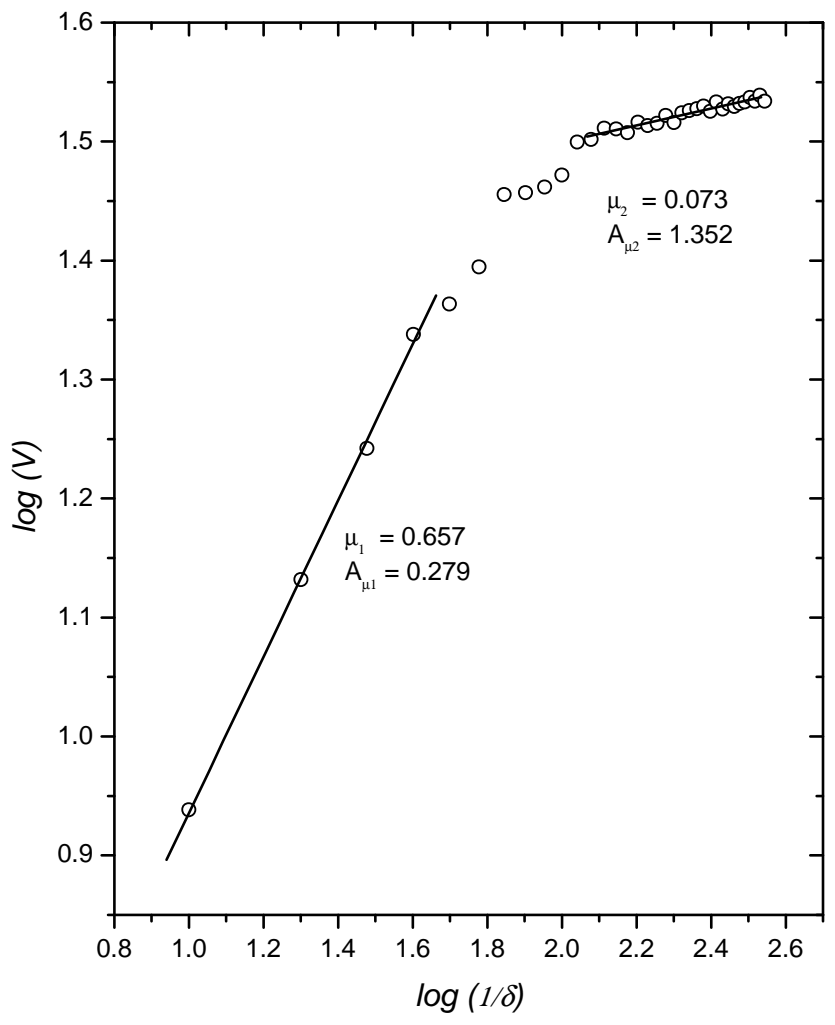

Fig. 3 


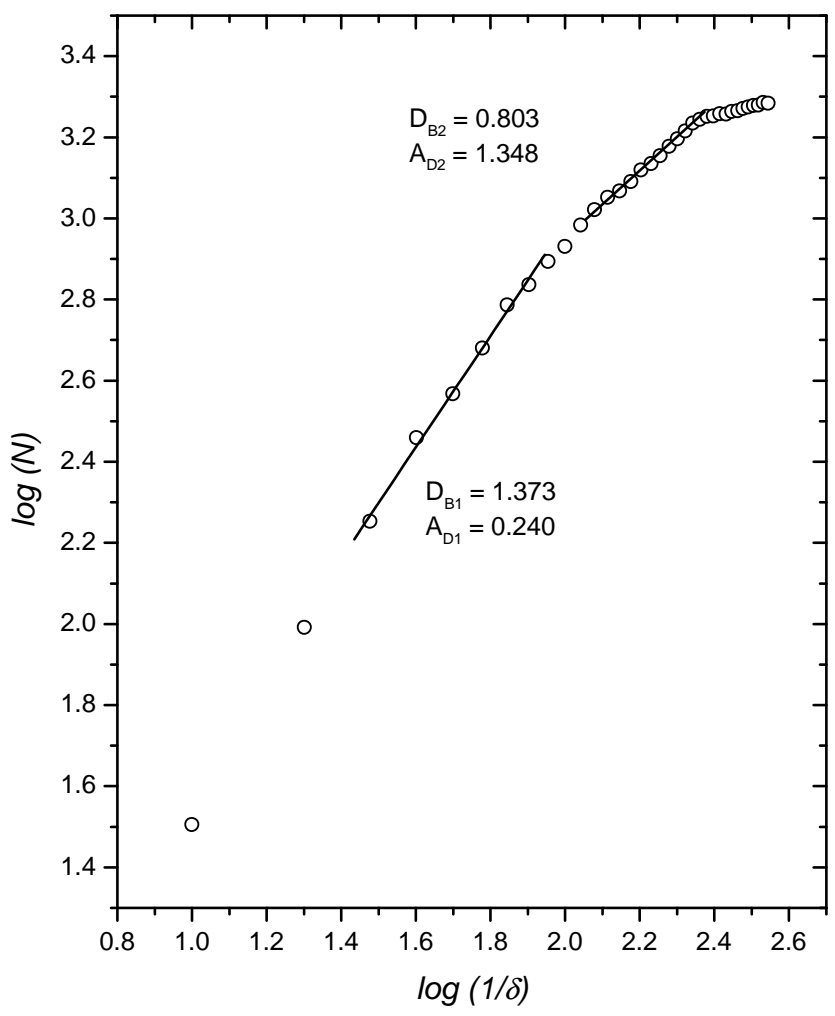

Fig. 4 


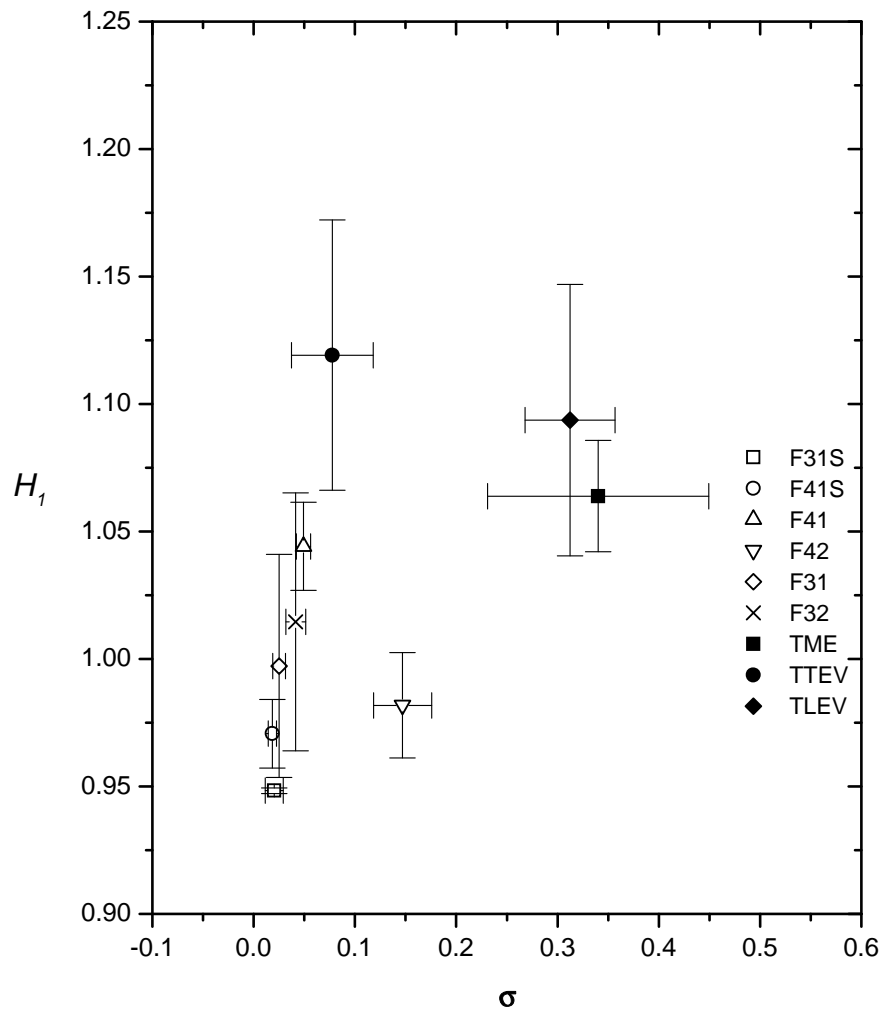

Fig. 5 


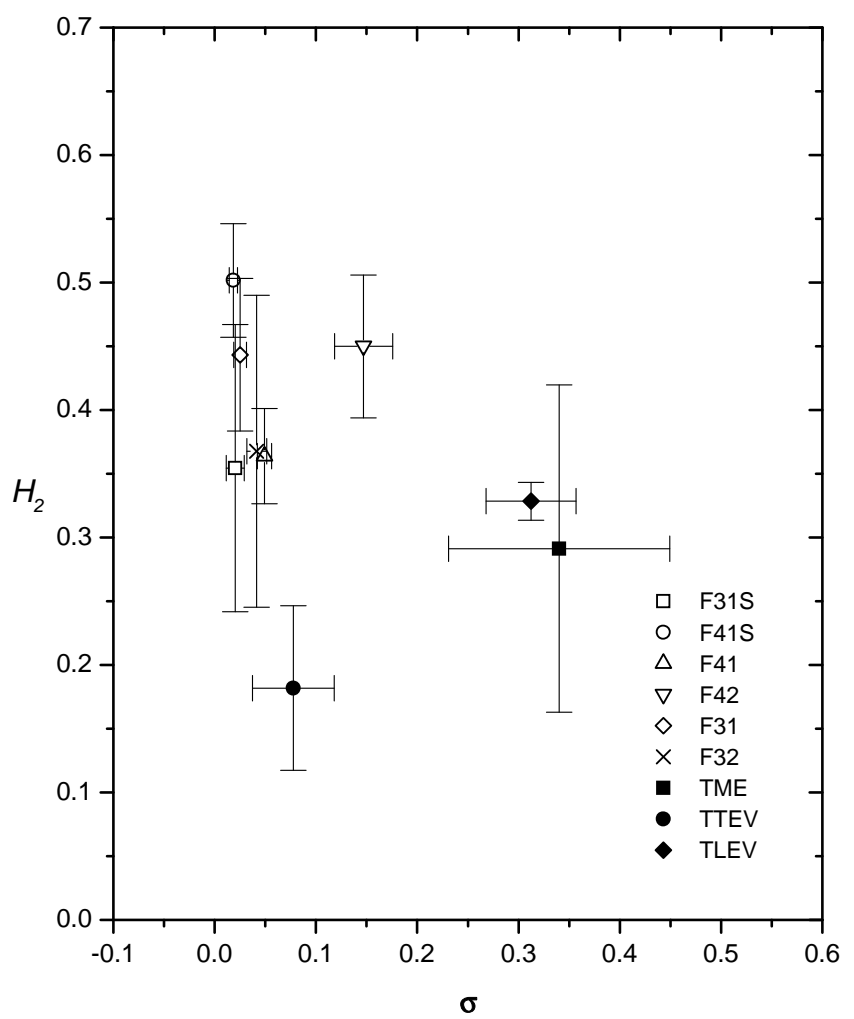

Fig. 6 


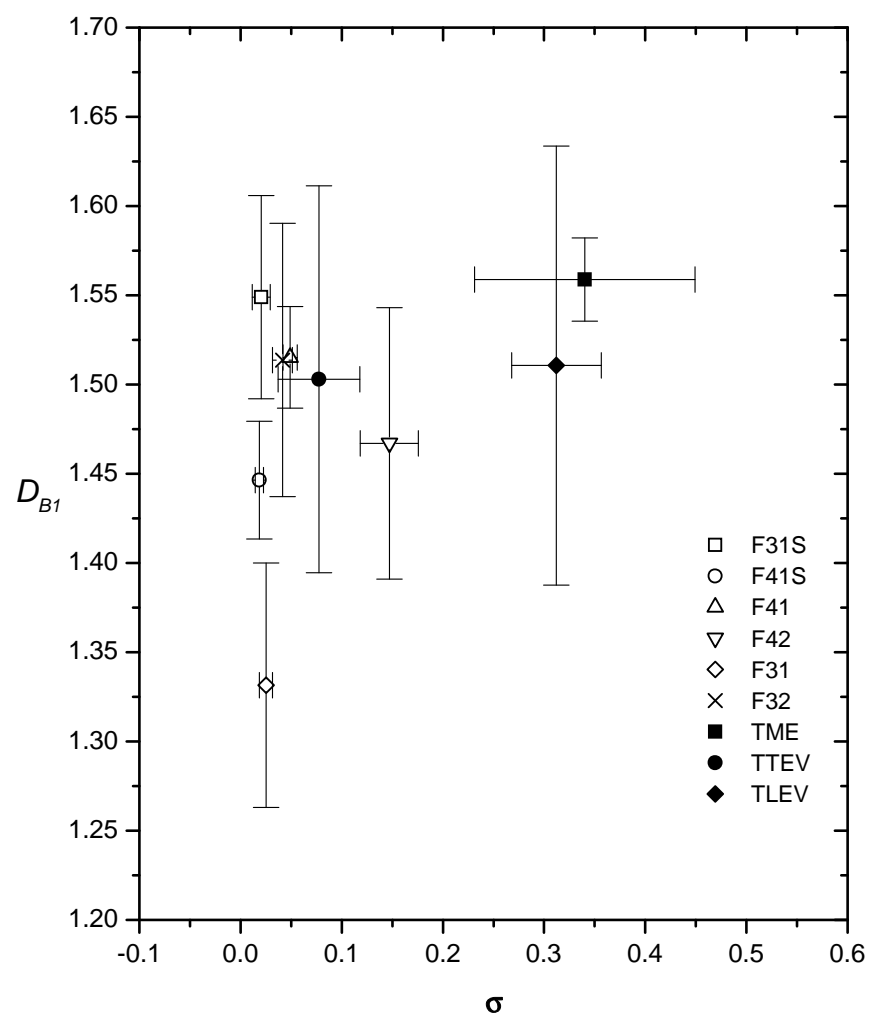

Fig. 7 


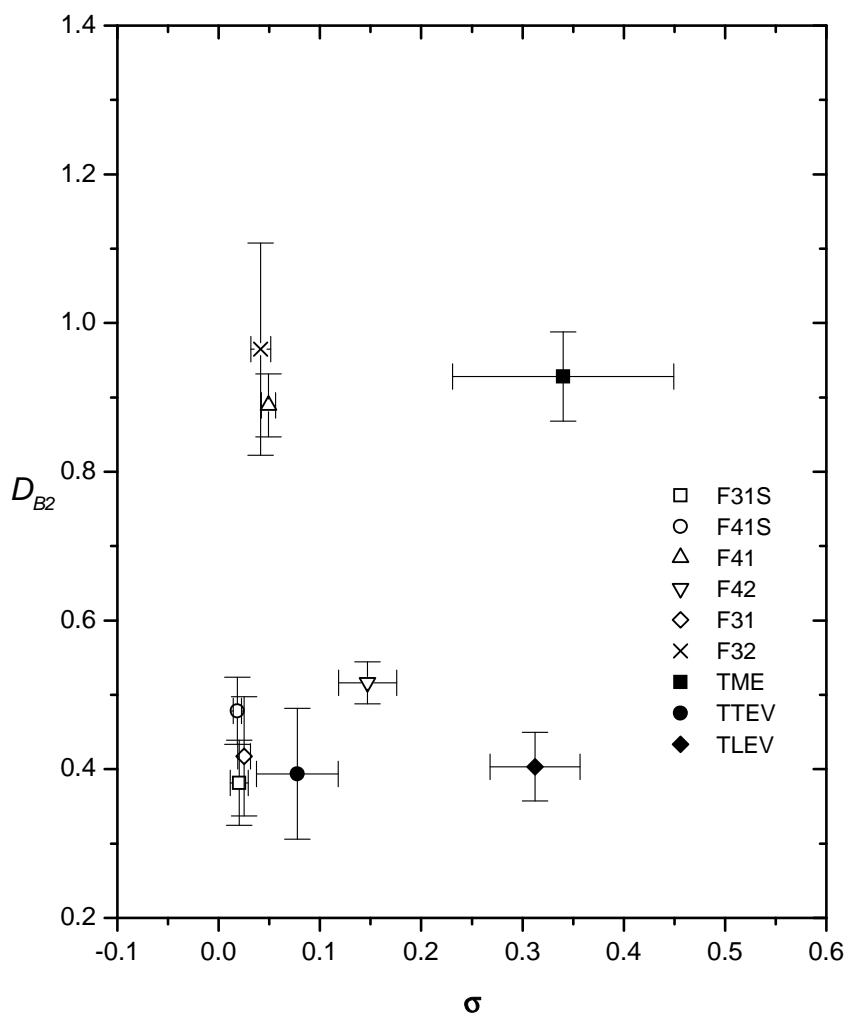

Fig. 8 


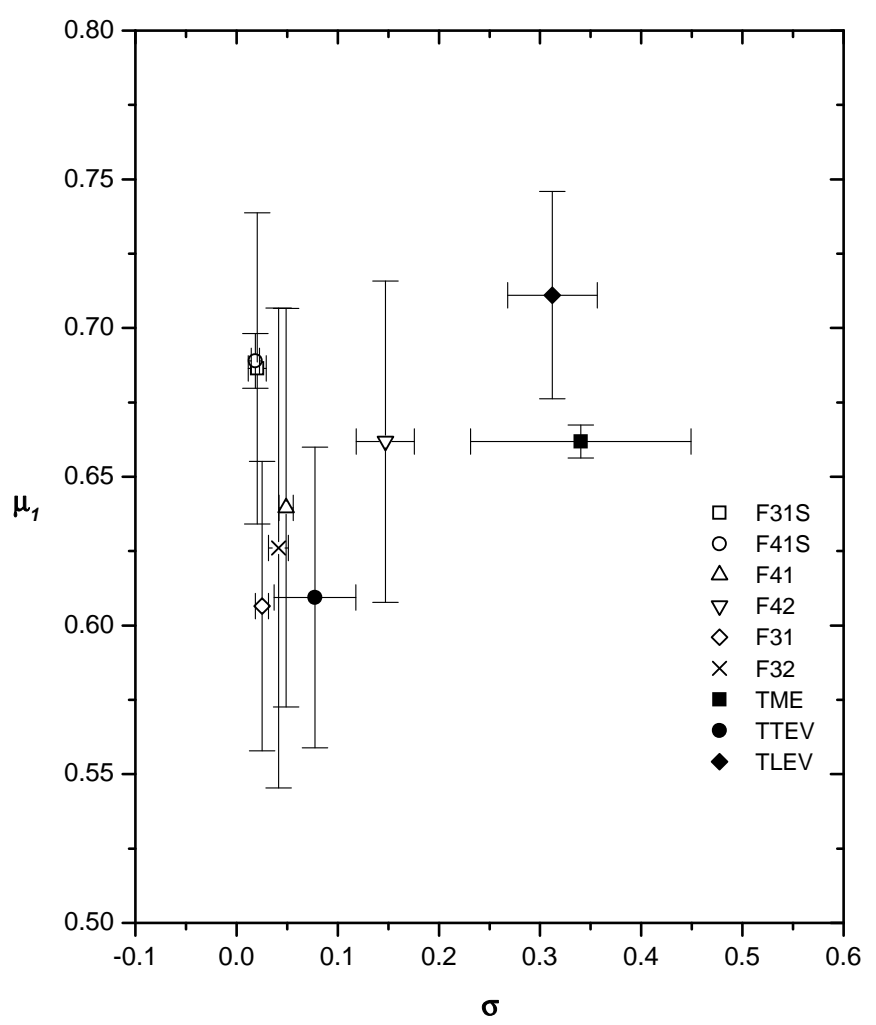

Fig. 9 


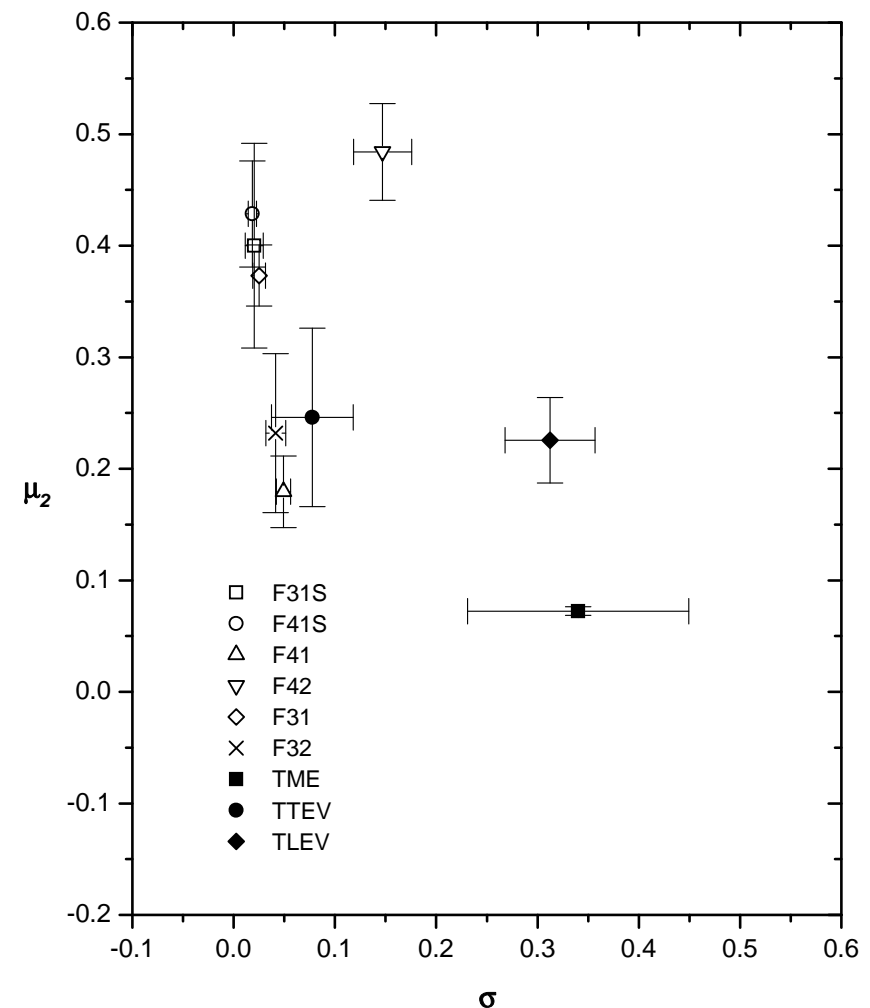

Fig. 10 


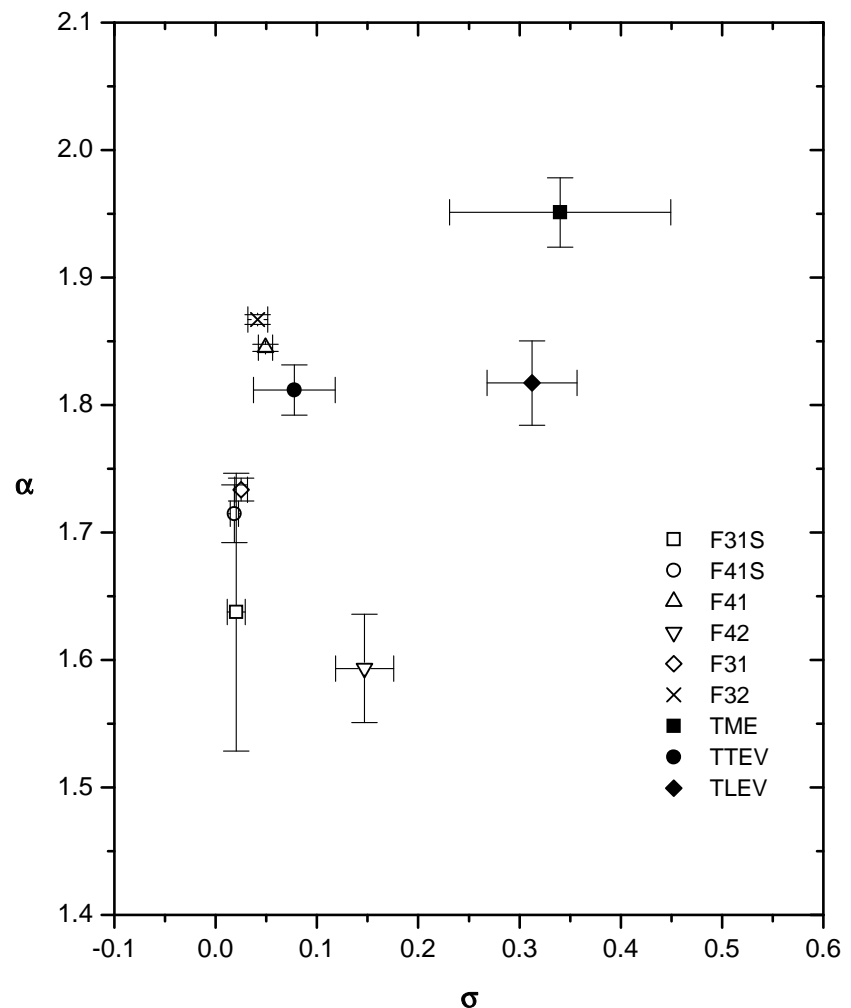

Fig. 11 


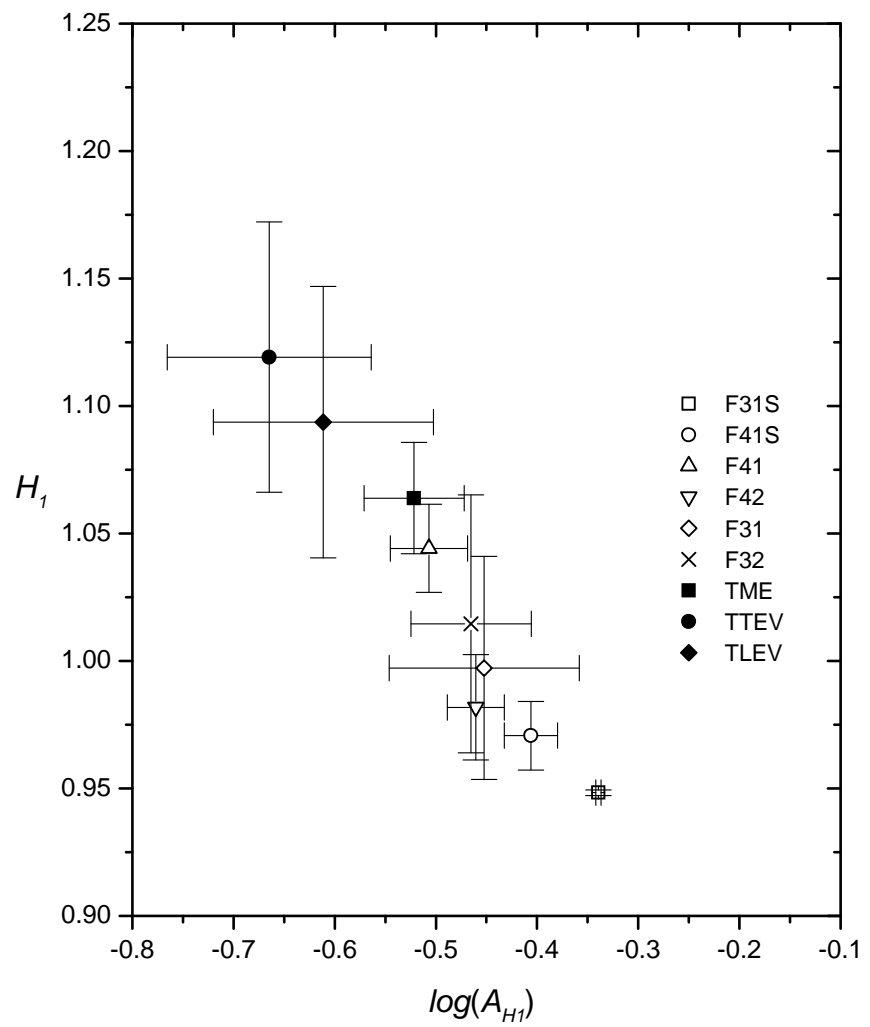

Fig. 12 


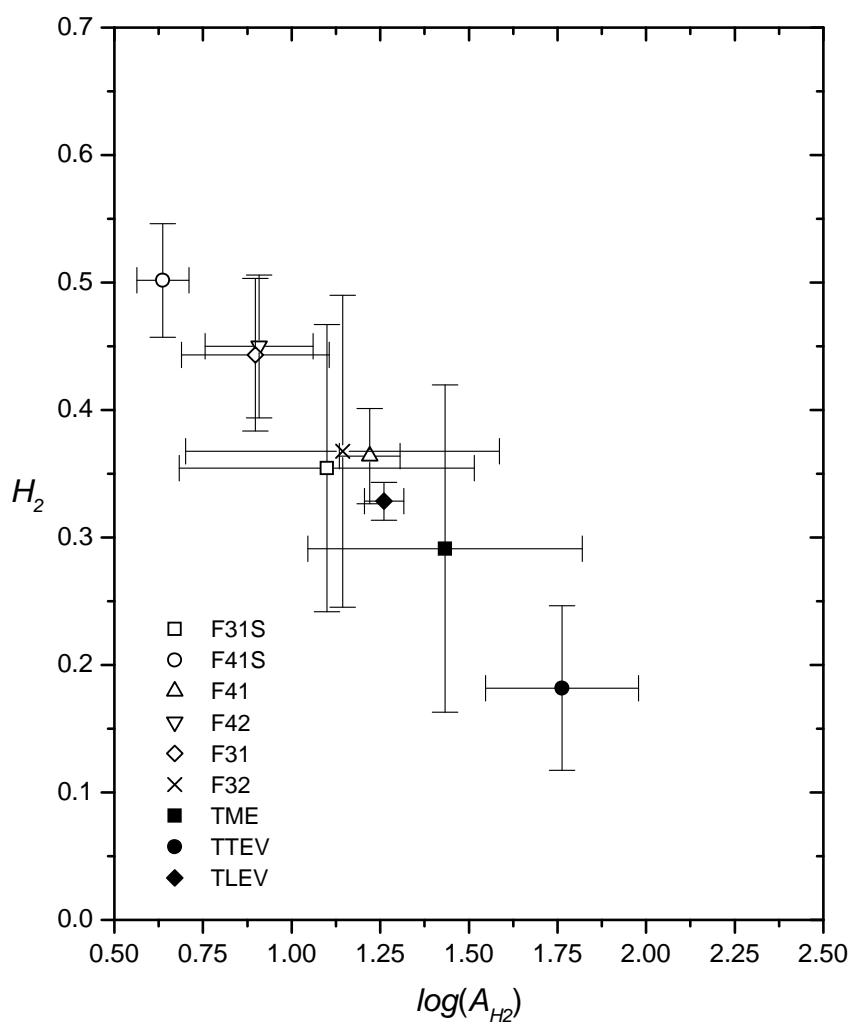

Fig.13 


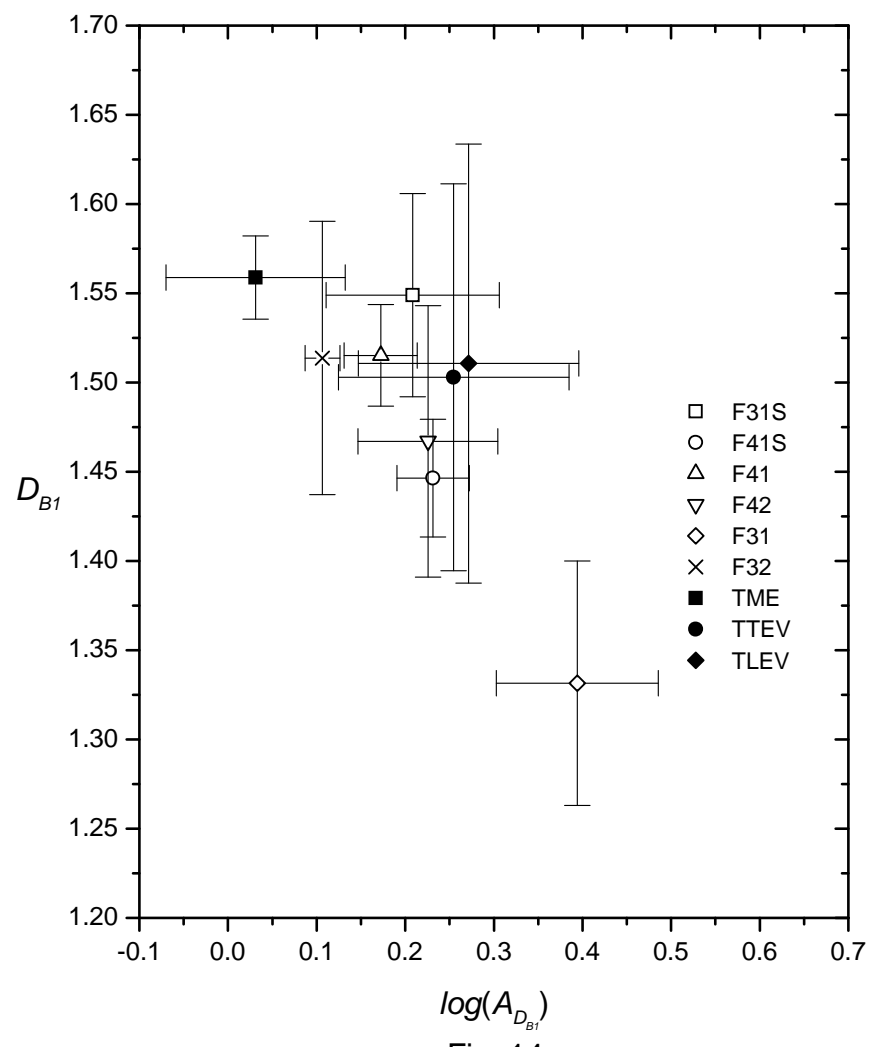

Fig. 14 


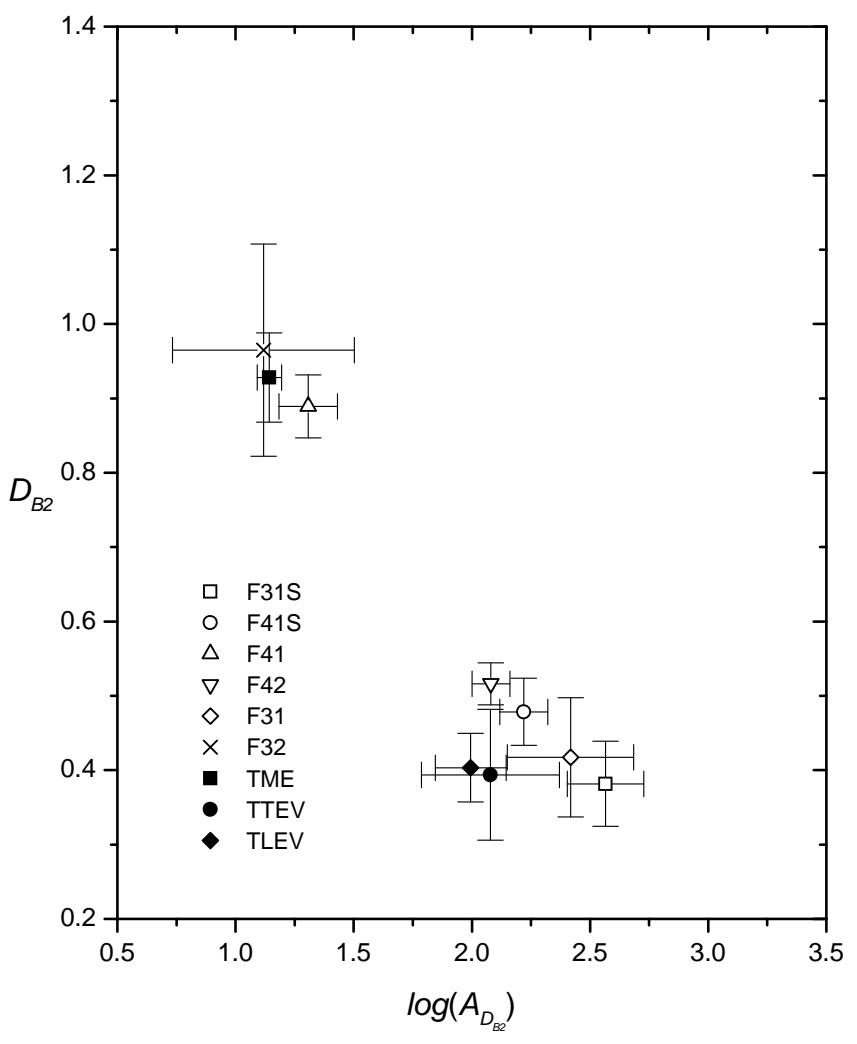

Fig.15 


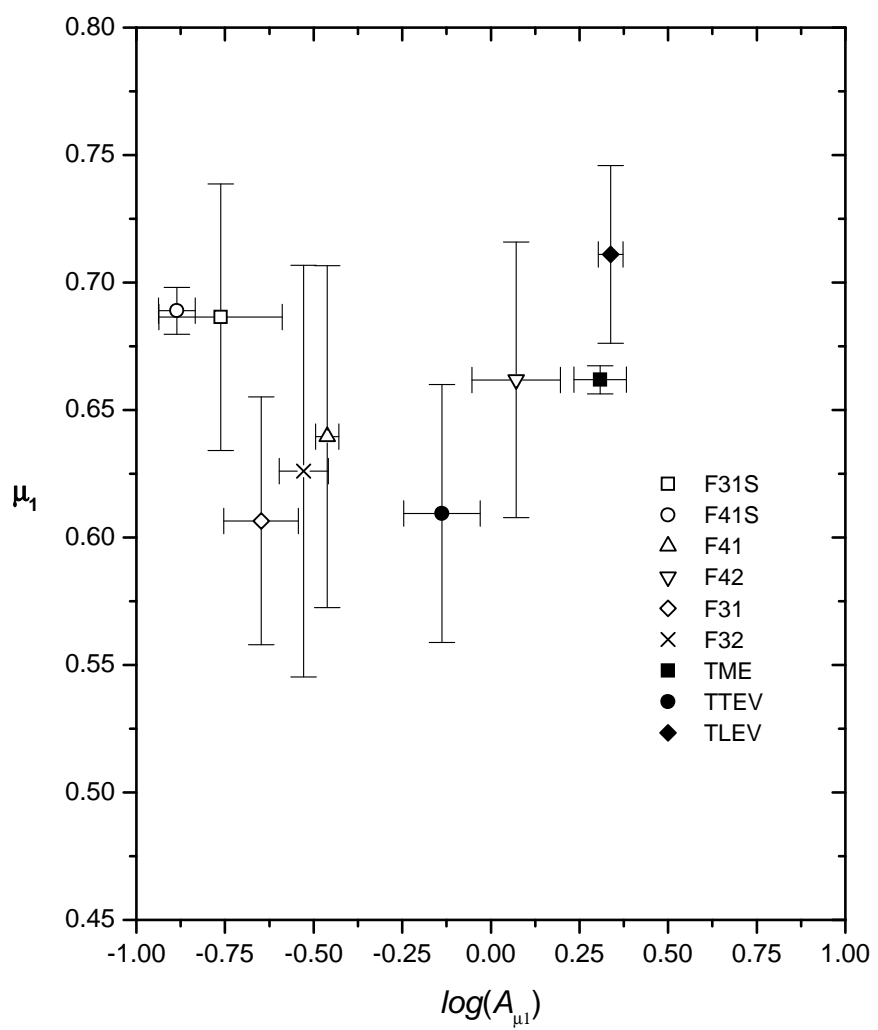

Fig. 16 


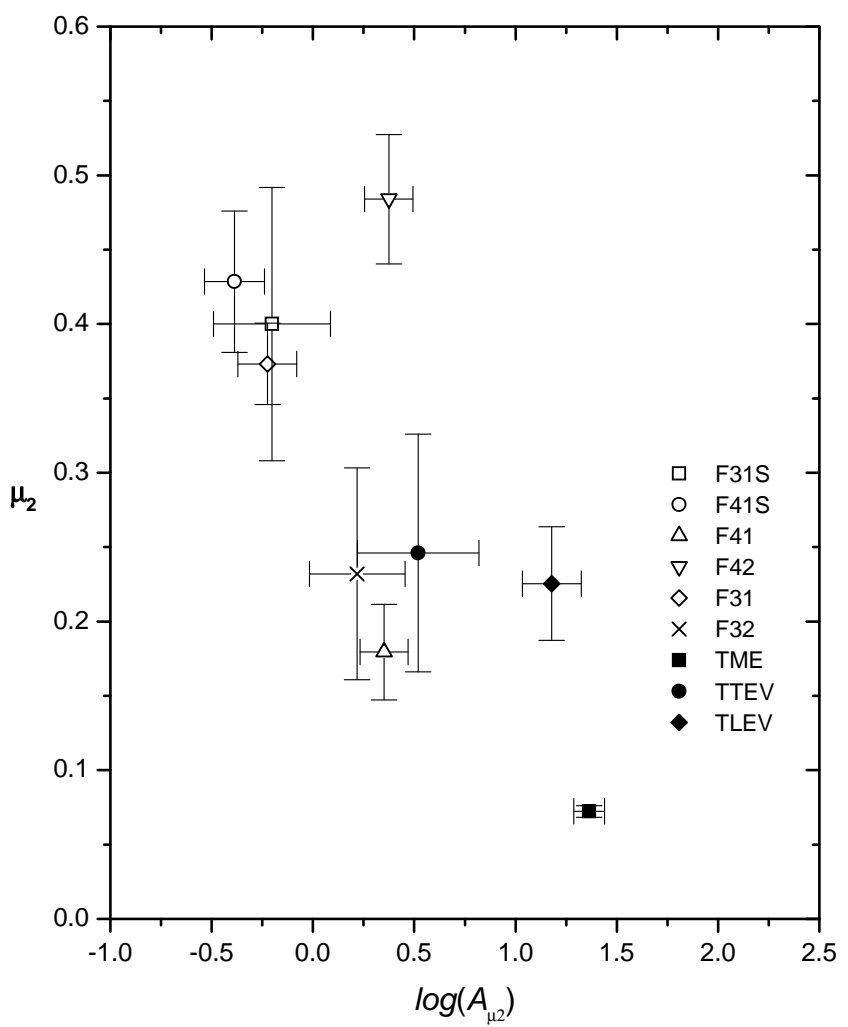

Fig. 17 


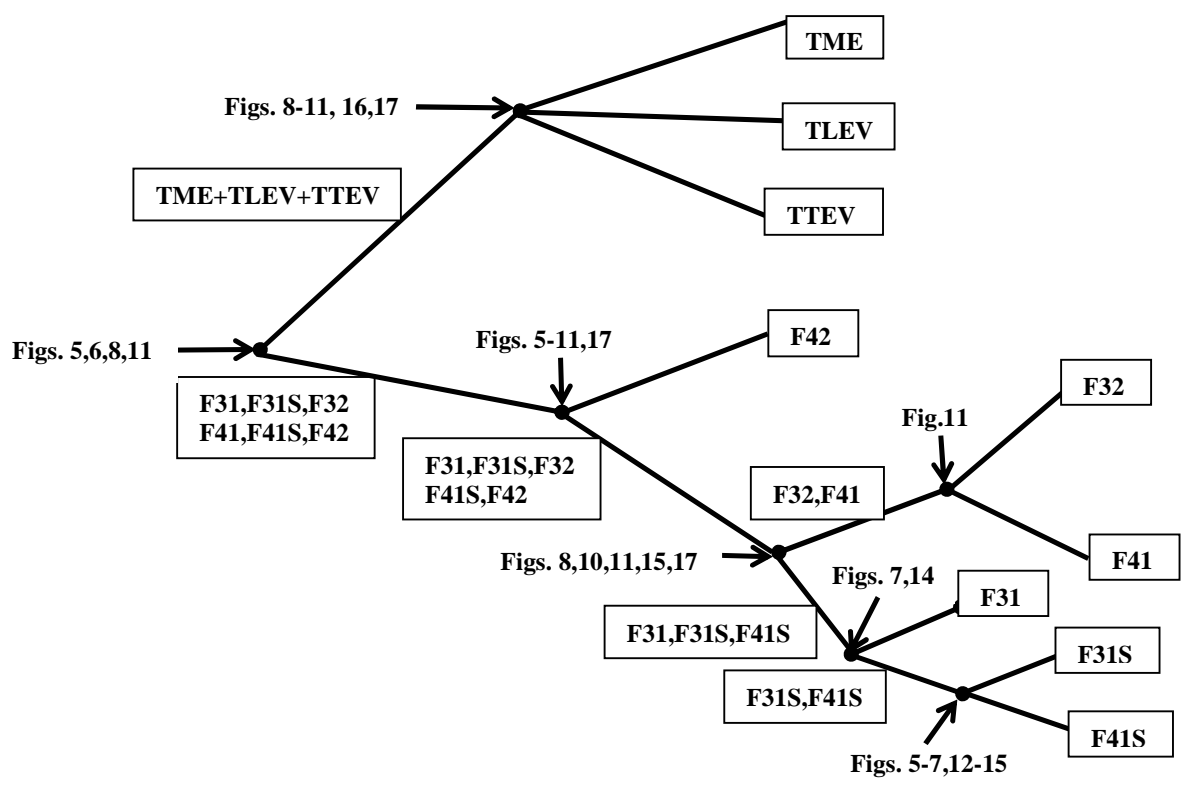

Fig. 18 